OPEN ACCESS

Edited by:

Min Zeng,

Lanzhou Institute of Chemical Physics

(CAS), China

Reviewed by:

Dongliang Chao,

University of Adelaide, Australia

Chao Wang,

Massachusetts Institute of

Technology, United States

*Correspondence:

Mengqiang Wu

mwu@uestc.edu.cn

Yuesheng Wang

wang.yuesheng@ireq.ca

Zaghib Karim

Zaghib.Karim@hydro.qc.ca

these authors have contributed

equally to this work

Specialty section:

This article was submitted to

Electrochemistry,

a section of the journal

Frontiers in Chemistry

Received: 08 July 2019 Accepted: 05 September 2019

Published: 02 October 2019

Citation:

Liu J, Xu Z, Wu M, Wang Y and Karim Z (2019) Capacity Contribution Induced by Pseudo-Capacitance Adsorption Mechanism of Anode

Carbonaceous Materials Applied in

Potassium-ion Battery.

Front. Chem. 7:640.

doi: $10.3389 /$ fchem.2019.00640

\section{Capacity Contribution Induced by Pseudo-Capacitance Adsorption Mechanism of Anode Carbonaceous Materials Applied in Potassium-ion Battery}

\author{
Jiahao Liu ${ }^{1 \dagger}$, Ziqiang $\mathrm{X} u^{1 \dagger}$, Mengqiang $W u^{1 *}$, Yuesheng Wang ${ }^{2 *}$ and Zaghib Karim ${ }^{2 *}$ \\ ${ }^{1}$ School of Materials and Energy, University of Electronic Science and Technology of China, Chengdu, China, ${ }^{2}$ Center of \\ Excellence in Transportation Electrification and Energy Storage, Hydro-Québec, Varennes, QC, Canada
}

The intrinsic bottleneck of graphite intercalation compound mechanism in potassium-ion batteries necessitates the exploitation of novel potassium storage strategies. Hence, utmost efforts have been made to efficiently utilize the extrinsic pseudo-capacitance, which offers facile routes by employing low-cost carbonaceous anodes to improve the performance of electrochemical kinetics, notably facilitating the rate and power characteristics for batteries. This mini-review investigates the methods to maximize the pseudo-capacitance contribution based on the size control and surface activation in recent papers. These methods employ the use of cyclic voltammetry for kinetics analysis, which allows the quantitative determination on the proportion of diffusion-dominated vs. pseudo-capacitance by verifying a representative pseudo-capacitive material of single-walled carbon nanotubes. Synergistically, additional schemes such as establishing matched binder-electrolyte systems are in favor of the ultimate purpose of high-performance industrialized potassium-ion batteries.

Keywords: potassium-ion batteries, carbonaceous anodes, pseudo-capacitance adsorption, surface doping activation, kinetic analysis

\section{INTRODUCTION}

Suffering from the geopolitical maldistribution of lithium resources, sodium-ion batteries (SIBs) and potassium-ion batteries (PIBs) reach a hotspot in view of wider resource reserves compared with lithium-ion batteries (LIBs) (2.09 wt\% of K vs. $2.36 \mathrm{wt} \%$ of $\mathrm{Na}$ vs. $0.0017 \mathrm{wt} \%$ of $\mathrm{Li}$ ) (Carmichael, 1989; Larcher and Tarascon, 2015). Significantly, the PIB system has the lowest negative potential $\left(0.15 \mathrm{~V}\right.$ below the $\left.\mathrm{Li} / \mathrm{Li}^{+}\right)$(Komaba et al., 2015; Eftekhari et al., 2016; Wang et al., 2018a,b) and satisfactory electrochemical kinetics in ionic diffusion kinetics and conductivity theoretically (Okoshi et al., 2013, 2017; Komaba et al., 2015; Eftekhari et al., 2016; Su et al., 2016) in non-aqueous electrolytes, ascribed to low de-solvation due to its weak Lewis acid character (Okoshi et al., 2017; Lei et al., 2018). Similar to the behavior of LIBs in graphite (Wang, 2017), the intercalation mechanism of PIBs involves three potassiation stages, generating the $\mathrm{KC}_{36}$ in Stage III, $\mathrm{KC}_{24}$ in Stage II, and finally the $\mathrm{KC}_{8}$ in Stage I (Jian et al., 2017) with $270 \mathrm{~mA} \mathrm{~h}$ $\mathrm{g}^{-1}$, which is far more stable than SIBs (Wang et al., 2013; Zheng et al., 2017). Nevertheless, large Shannon ionic radius $\left(\mathrm{K}^{+}=1.38 \AA, \mathrm{Na}^{+}=1.02 \AA, \mathrm{Li}^{+}=0.76 \AA\right)$ and atomic mass 
$(\mathrm{K}=39.10, \mathrm{Na}=22.99, \mathrm{Li}=6.94) \quad$ (Shannon, 1976) have decreased the theoretical capacity and induced high volume expansion of 61\% (Wen et al., 2015; Eftekhari et al., 2016; Zou et al., 2017). Although some strategies enter into consideration such as adopting expand graphite (An et al., 2018), implementing solvent co-intercalation (SCI) (Wang et al., 2013, 2019; David and Singh, 2014), and developing dual-carbon batteries (DCBs) (Carlin et al., 1996, 2010; Beltrop et al., 2017; Fan et al., 2017; Ji et al., 2017), essential kinetics deficiency is hard to surmount.

To address the irreversible expansion induced by equilibrium graphite, amorphous carbons come into the focused sight (Xing et al., 2017), which are admittedly classified as hard carbon (HC) and soft carbon (SC). HC is proven to have a prolonged cycling ability for its randomly oriented bend graphitic layers along the c axis without observed expansion after a thorough potassiation (Jian et al., 2016, 2017). On the contrary, SC is easily graphitized with turbostratic domains although far from the commercial graphite. SC presents obvious expansion to $\mathrm{HC}$ if undergoing complete potassiation (Luo et al., 2015a; Wang et al., 2017). Nonetheless, SC has a better rate performance than HC for more aligned domains (Jian et al., 2017), regarded as the reason for the better rate performance.

Pseudo-capacitance is the middle part of the battery and electrical double-layer capacitors (EDLCs) (Jiang and Liu, 2019) as shown in Figure 1a. In Figure 1a, Liu points out that the current generation of batteries depends on the Faradic electron transfer from the surface to the metal center based on the chargecompensating ions by intercalation or adsorption. In contrast, a pseudo-capacitor is different from EDLCs because it is not electrostatic-induced and the transfer process of surface electrons distinguishes the behavior from batteries. Pseudo-capacitance can be classified into two categories-intrinsic and extrinsic. The former (Chao et al., 2016) describes an inherent feature of specific materials such as $\mathrm{RuO}_{2}$ and $\mathrm{MnO}_{2}$, which is on the strength of Faradaic electron transfer. However, the latter emphasizes the technological means of low dimension, nanoscale size, and high surface area (Wang et al., 2007; Brezesinski et al., 2009; Muller et al., 2015; Cook et al., 2016) among a majority of materials, for instance, the single-walled carbon nanotube (SWCNT) with surface-enriched potassium ions in Figure 1b (Hersam, 2009; Kang et al., 2013), attributed to the regular hexagonal arrangement of carbon atoms on the surface. This non-Faradaic pathway provides a possibility to utilize inexpensive carbonaceous anodes (Gogotsi and Penner, 2018) if proper surface treatments such as activization, doping, and plasma processing have been undergone (Chao et al., 2018).

These surface-dominated anodes uptake and absorb potassium ions with fast reaction kinetics during the electrochemistry process, avoiding the hindrance in the intercalation mechanism, which is regarded as the primary cause for high rate property and large capacity of PIBs undergoing charging and discharging processes (Shin et al., 2011; Shao et al., 2013; Chen et al., 2016; Long et al., 2016). As a consequence, this mini-review analyzes the methods for distinguishing the proportion of capacity contribution and summarizes the application of pseudo-capacitance to PIBs very recently, aiming to design a practical performance improvement approach.

\section{ANALYSES AND APPLICATIONS OF PSEUDO-CAPACITANCE}

Generally, it is widely admitted that pseudo-capacitance is not a pure Faradaic progress but a rapid reversible surface redox reaction involved in EDLCs. The charge storage mechanism of complex PIBs behaviors is composed of two typical contribution progresses: surface-induced pseudo-capacitor process and diffusion-dominated process (Brezesinski et al., 2009; Wen et al., 2015; Xu et al., 2019b). Nonetheless, Faradaic and non-Faradaic reactions are electro indistinguishable for jointly contributing to the current parameter (Gogotsi and Penner, 2018). Most researchers employ cyclic voltammetry $(\mathrm{CV})$ to determine the relative proportion of contribution from pseudo-capacitor and diffusion-dominated processes. The peak current is proportional to the square root of sweep rate describing the reversible diffusion-limited state $\left(i-v^{\frac{1}{2}}\right)$, whereas it is proportional to the sweep rate $(i-v)$ describing the capacitive state. A representative power law relationship between the current and scan rate reveals the charge storage mechanism in PIBs (Wang et al., 2007; Torsten et al., 2010; Veronica et al., 2013):

$$
\mathrm{i}=\mathrm{a} v^{b}
$$

where $a$ and $b$ are constants. The $b$ value can be figured out by profiling the $\log (i)-\log (v)$ curve. If $b=0.5$, the Faradic diffusion is predominant; while $b=1$, the pseudo-capacitance assumes the primary contribution (Sathiya et al., 2011; Lijun et al., 2014; Zou et al., 2017). Furthermore, as for a fixed sweep rate, the specific pseudo-capacitance contribution can be given in detail by the following formula (Torsten et al., 2010; Wang Y. et al., 2016):

$$
\mathrm{i}=k_{1} v+k_{2} v^{\frac{1}{2}}
$$

where the parameter $k_{1} v$ represents the capacitive process while the $k_{2} v^{\frac{1}{2}}$ is in favor of the diffusion process as stated earlier.

Furthermore, Marveh maintains that compared with the CV method, the step potential electrochemical spectroscopy (SPECS) has wider adaptive range with prominent advantages. In high sweep rates, SPECS presents precise characterization to depict the process of electrical double layer on the surface of electrodes (Forghani and Donne, 2018).

Recent works validate the validity of the pseudo-capacitance algorithm based on the surface-dominated pseudo-capacitance mechanism, which has been extensively applied in carbonaceous anodes in PIBs by constructing high surface area or activating.

Doping and activating are highly feasible methods that introduce abundant defects, expand specific surface area, and promote the conductivity, meanwhile adding charge storage for PIBs (Lijun et al., 2014; Share et al., 2016; Chen et al., 2017; Lei et al., 2018; Xu et al., 2018).

Nitrogen-doped strategy has a practical significance eliciting satisfying performance enhancements. According to the X-ray photoelectron spectroscopy (XPS) results, pyrrole nitrogen (N5 ), pyridine nitrogen (N-6), and quaternary $\mathrm{N}(\mathrm{N}-\mathrm{Q})$ are three $\mathrm{N}$ doping forms presented in Figure 2A, where N-5 and N-6 possess high electrochemical activity and generate additional defects in the surface of the graphene layer, hence promoting the adsorption 

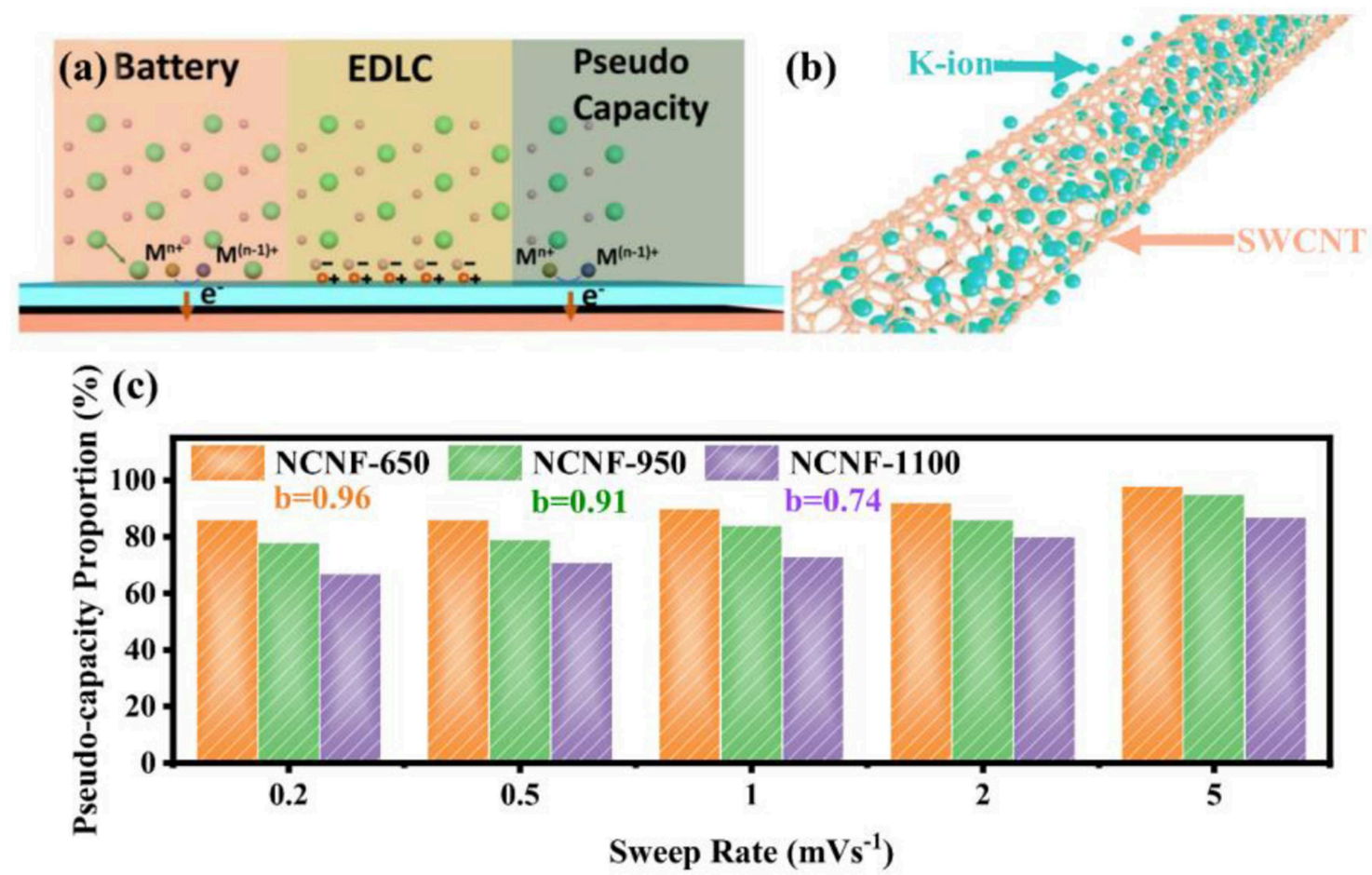

FIGURE 1 | (a) Surface processes among battery, EDLC and pseudo-capacitance. (b) Diagram of the surface-dominated procedure for SWCNT; with permission from Wiley. (c) Pseudo-capacity proportion of NCNF-650, NCNF-950 and NCNF-1100 with b value; with permission from Springer.
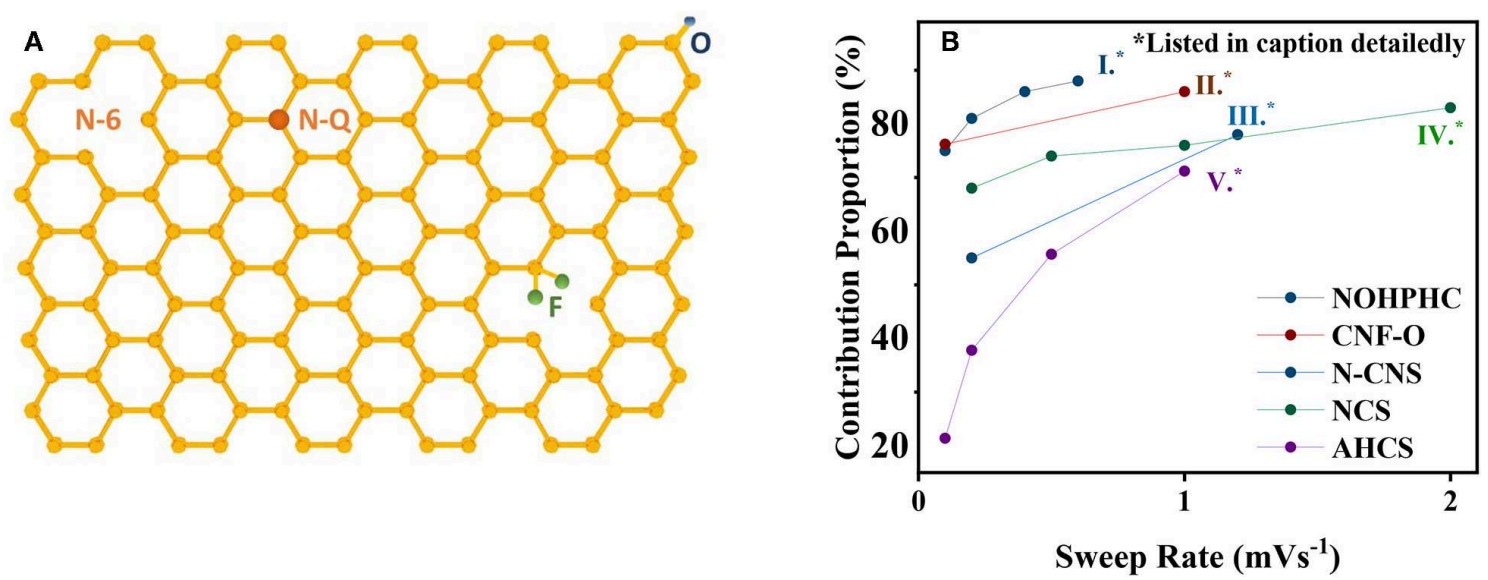

FIGURE 2 | (A) Surface defects induced by N-doping, O-doping and F-doping. (B) Relationship between pseudo-capacitance contribution proportion and sweep rate based on recent researches (I) (Yang et al., 2018), (II) (Adams et al., 2017), (III) (Lei et al., 2018), (IV) (Chen et al., 2017), (V) (Wang et al., 2018).

quantity of potassium ions, accelerating the kinetic process ( $\mathrm{Li}$ et al., 2013; Wang et al., 2014; Xu et al., 2018). This differentiation of $\mathrm{N}$ forms is ascribed to their constructions of respective bonding electrons, resulting in different chemical activities. However, N-Q, located in the internal surface of graphene layer, bonding with three sp2 carbon atoms, is beneficial to improve electrical conductivity (Yang et al., 2018). Notably, N-6 is regarded as the most effective doping precursor because it replaces the carbon atom with a nitrogen atom at the defect or the edge of the graphite plane and occupies abundant active centers to adsorb potassium ions (Ma et al., 2012; Ding et al., 2014; Xie et al., 2017). Consistently, recent researches demonstrated that N-6 defects decreases with temperature increasing; meanwhile, the degree of graphitization rises, accompanied by the generation of N-Q. Xu concludes that among three temperature-controlled materials NCNF-650, NCNF-950, and NCNF-1100 derived from poly-pyrrole nanofibers, the pseudo-capacitance contribution of NCNF-650 occupies $90 \%$ at $1 \mathrm{mV} \mathrm{s}^{-1}$ for abundant N-6 defects, while the others occupy 73 and $84 \%$ at $1 \mathrm{mV} \mathrm{s}^{-1}$ as displayed in Figure 1c with their $b$ value ( $\mathrm{Xu}$ et al., 2018). The 
$b$ value increases with the temperature dropping, revealing the degree deepening of pseudo-capacity, in accordance with the quantity N-6 defects. Similar results are obtained in Xie's report; nonetheless, Xie indicates that enhancement of electrochemical performance is a comprehensive result associated with N-6 defects, electrical conductivity, and transfer resistance. Three sets of temperature-controlled experiments point out that PNCM700 is equipped with the best comprehensive performance compared with PNCM-500 and PNCM-900. As for the function of defects, Clement states that the D-bond from the Raman spectrum is employed to describe the $\mathrm{sp}^{3}$ defect distribution, which intensifies a six-fold rate performance to the un-doped material (Clement et al., 2015; Share et al., 2016). The prominent significance of $\mathrm{N}$-doping is to spread out the interlayer spacing and provide huge specific surface area to promote the pseudocapacitive effect.

Doping some other elements also achieves fair results. Oxidation functional groups on the carbon surface polishes up the wettability of carbon-based materials and advances the pseudo-capacitance behavior (Tarun et al., 2010; Shao et al., 2013; Wang X. et al., 2016; Wu et al., 2016; Xie et al., 2017; Wang et al., 2018). Adams reported that oxidation groups increase obviously on the surface capacitive storage while inducing the capacity reduction contributed by the intercalation mechanism. As a consequence, there is no significant enhancement to the total capacity (Adams et al., 2017). In addition, mixed-doping $\mathrm{P}$ and $\mathrm{O}$ doping (Ma et al., 2017) based on the triphenylphosphine precursor obtains a satisfactory capacity of $474 \mathrm{~mA} \mathrm{~h} \mathrm{~g}^{-1}$, benefiting from expanding the interlayer spacing; $\mathrm{N}$ and $\mathrm{F}$ doping immensely adds the conductivity distinctly (Ju et al., 2016; Share et al., 2016; Adams et al., 2017).

Activated hollow carbon nanospheres (HCS) underwent HF etching from C@SiO2 nanospheres in Wang's work. Wang emphasizes on the sharp increase on the surface area from 481.4 to $757.8 \mathrm{~m}^{2} \mathrm{~g}^{-1}$ after activating utilizing $\mathrm{KOH}$ as the activator. Capacitive contribution occupies $71.2 \%$ at a sweep rate of $1 \mathrm{mV}$ $\mathrm{s}^{-1}$, leading to $192.7 \mathrm{~mA} \mathrm{~h} \mathrm{~g}^{-1}$ at $2 \mathrm{~A} \mathrm{~g}^{-1}$ after 5,000 cycles with a retention of $99.5 \%$ (Wang et al., 2018). Aforesaid data support the rule that the pseudo-capacity contribution has the tendency of positive correlation with sweep rate as summarized in Figure 2B. This work claimed that activated hollow carbon expands the layer spacing of the carbon anode and shortens the diffusion distance of K-ions.

Nevertheless, surface-modified strategies, whether doping or activating, may give rise to the decrease of initial Coulombic

\section{REFERENCES}

Adams, R. A., Syu, J. M., Zhao, Y., Lo, C. T., Varma, A., and Pol, V. G. (2017). Binder-free N- and O-rich carbon nanofiber anodes for long cycle life K-ion batteries. ACS Appl. Mater. Interfaces 9, 17872-17881. doi: 10.1021/acsami.7b02476

An, Y., Fei, H., Zeng, G., Ci, L., Xi, B., Xiong, S., et al. (2018). Commercial expanded graphite as a low-cost, long-cycling life anode for potassium-ion batteries with conventional carbonate electrolyte. J. Power Sources 378, 66-72. doi: 10.1016/j.jpowsour.2017.12.033 efficiency (ICE) unsatisfactorily. Compensatory methods work well in LIBs and SIBs (Suo et al., 2017) to establish appropriate binder-electrolyte systems (BESs), which directly impact the formation of solid electrolyte interphase (SEI), especially the morphology features such as thickness, pore, and wrinkle. Tailored BESs shape the SEI into a smooth and thin layer, hence improving the transfer efficiency of ions on the phase interface (Xu et al., 2019a). Similarly, employing KFSI and KTFSI electrolyte (Eftekhari et al., 2016; Jin et al., 2016), adding electrolyte additives (Wu et al., 2017), selecting hydrophilic binders such as CMC, PANa, and SA (Komaba et al., 2015; Luo et al., 2015b; Jin et al., 2016; Xu et al., 2019a), and utilizing prepotassiation technique (Yang et al., 2018) serve the same purpose for superb PIBs.

\section{CONCLUSIONS AND PERSPECTIVES}

PIBs with carbonaceous anodes provide the possibility for industrialization under controlled price. Facilely, surface modifications such as doping and activating obviously enhance the pseudo-capacitance contribution, speeding up the rate and power performance based on a rapid electrochemical kinetics.

This non-insertion charge storage (pseudo-capacitance absorption) integrates with both battery-type and capacitortype characteristics, exhibiting distinct redox separation peaks including analogous linear capacitive voltage response. However, the relationship between capacitive and sweep rate is only authentic limited in a low and narrow sweep rate under the CV separation method. Precisely, the SPECS is suitable for a wider range of sweep rates, inducing detailed contribution information for each potential point (Forghani and Donne, 2018). In addition, matching binder-electrolyte with anodes accurately can synergistically promote specific capacity and rate properties, deriving high-performance PIBs.

\section{AUTHOR CONTRIBUTIONS}

All authors listed have made a substantial, direct and intellectual contribution to the work, and approved it for publication.

\section{FUNDING}

This work was supported by the Sichuan Science and Technology Program (Grant Nos. 2018GZ0006 and 2018GZ0134).

Beltrop, K., Beuker, S., Heckmann, A., Winter, M., Placke, T. J. E., and Science, E. (2017). Alternative electrochemical energy storage: Potassiumbased dual-graphite batteries. Energy Environ. Sci. 10, 2090-2094. doi: 10.1039/C7EE01535F

Brezesinski, T., Wang, J., Polleux, J., Dunn, B., and Tolbert, S. H. (2009). Templated nanocrystal-based porous $\mathrm{TiO}(2)$ films for next-generation electrochemical capacitors. J. Am. Chem. Soc. 131, 1802-1809. doi: 10.1021/ja80 57309

Carlin, R. T., Fuller, J., Kuhn, W. K., Lysaght, M. J., and Trulove, P. C. (1996). Electrochemistry of room-temperature chloroaluminate molten salts 
at graphitic and nongraphitic electrodes. J. Appl. Electrochem. 26, 1147-1160. doi: 10.1007/BF00243740

Carlin, R. T., Long, H. C. D., Fuller, J., and Trulove, P. C. (2010). ChemInform abstract: dual intercalating molten electrolyte batteries. Cheminform 25, L73L76. doi: 10.1002/chin.199446010

Carmichael, R. S. (1989). CRC Practical Handbook of Physical Properties of Rocks and Minerals. Boca Raton, FL: CRC Press.

Chao, D., Ouyang, B., Liang, P., Huong, T. T. T., Jia, G., Huang, H., et al. (2018). C-plasma of hierarchical graphene survives SnS bundles for ultrastable and high volumetric Na-ion storage. Adv. Mater. 30:1804833. doi: 10.1002/adma.201804833

Chao, D., Zhu, C., Yang, P., Xia, X., Liu, J., Wang, J., et al. (2016). Array of nanosheets render ultrafast and high-capacity Na-ion storage by tunable pseudocapacitance. Nat. Commun. 7:12122. doi: 10.1038/ncomms12122

Chen, C., Wang, Z., Zhang, B., Miao, L., Cai, J., Peng, L., et al. (2017). Nitrogen-rich hard carbon as a highly durable anode for high-power potassium-ion batteries. Energy Storage Mater. 8, 161-168. doi: 10.1016/j.ensm.2017.05.010

Chen, C., Xu, H., Zhou, T., Guo, Z., Chen, L., Yan, M., et al. (2016). Integrated intercalation-based and interfacial sodium storage in graphene-wrapped porous $\mathrm{Li}_{4} \mathrm{Ti}_{5} \mathrm{O}_{12}$ nanofibers composite aerogel. Adv. Energy Mater. 6:1600322. doi: 10.1002/aenm.201600322

Clement, B., Todd Wesley, S., Michelle, D., and Xiulei, J. J. N. L. (2015). New mechanistic insights on Na-ion storage in nongraphitizable carbon. Nano Lett. 15, 5888-5892. doi: 10.1021/acs.nanolett.5b01969

Cook, J. B., Kim, H. S., Yan, Y., Ko, J. S., Robbennolt, S., Dunn, B., et al. (2016). Mesoporous $\mathrm{MoS}_{2}$ as a transition metal dichalcogenide exhibiting pseudocapacitive Li and Na-ion charge storage. Adv. Energy Mater. 6:1501937. doi: $10.1002 /$ aenm.201501937

David, L., and Singh, G. (2014). Reduced graphene oxide paper electrode: opposing effect of thermal annealing on $\mathrm{Li}$ and Na cyclability. J. Phys. Chem. C 118, 28401-28408. doi: 10.1021/jp5080847

Ding, N., Huang, Z. H., Lv, R., Lu, Y., Wang, J., Shen, W., et al. (2014). Nitrogenenriched electrospun porous carbon nanofiber networks as high-performance free-standing electrode materials. J. Mater. Chem. A 2, 19678-19684. doi: $10.1039 / C 4 T A 03868 \mathrm{~A}$

Eftekhari, A., Jian, Z., and Ji, X. (2016). Potassium secondary batteries. ACS Appl. Mater. Interfaces 9, 4404-4419. doi: 10.1021/acsami.6b07989

Fan, L., Liu, Q., Chen, S., Lin, K., Xu, Z., and Lu, B. J. S. (2017). Potassiumbased dual ion battery with dual-graphite electrode. Small 13:1701011. doi: 10.1002/smll.201701011

Forghani, M., and Donne, S. W. (2018). Method comparison for deconvoluting capacitive and pseudo-capacitive contributions to electrochemical capacitor electrode behavior. J. Electrochem. Soc. 165, A664-A673. doi: 10.1149/2.0931803jes

Gogotsi, Y., and Penner, R. M. (2018). Energy storage in nanomaterialsCapacitive, pseudocapacitive, or battery-like? Acs Nano 12, 2081-2083. doi: 10.1021/acsnano.8b01914

Hersam, M. C. (2009). "Progress towards monodisperse single-walled carbon nanotubes," in Nanoscience and Technology, ed P. Rodgers (London: Macmillan Publishers Ltd.), 3-10. doi: 10.1142/9789814287005 0001

Ji, B., Fan, Z., Wu, N., and Tang, Y. (2017). A dual-carbon battery based on potassium-ion electrolyte. Adv. Energy Mater. 7:1700920. doi: 10.1002/aenm.201700920

Jian, Z., Hwang, S., Li, Z., Hernandez, A. S., Wang, X., Xing, Z., et al. (2017). Hardsoft composite carbon as a long-cycling and high-rate anode for potassium-ion batteries. Adv. Funct. Mater. 27:1700324. doi: 10.1002/adfm.201700324

Jian, Z., Xing, Z., Bommier, C., Li, Z., and Ji, X. (2016). Hard carbon microspheres: potassium-ion anode versus sodium-ion anode. Adv. Energy Mater. 6:1501874. doi: 10.1002/aenm.201501874

Jiang, Y., and Liu, J. (2019). Definitions of pseudocapacitive materials: a brief review. Energy Environ. Sci. 2, 30-37. doi: 10.1002/eem2.12028

Jin, Z., Zou, X., Zhu, Y., Xu, Y., and Wang, C. (2016). Electrochemical intercalation of potassium into graphite. Adv. Funct. Mater. 26, 8103-8110. doi: $10.1002 / \mathrm{adfm} .201602248$

Ju, Z., Zhang, S., Xing, Z., Zhuang, Q., Qiang, Y., and Qian, Y. (2016). Direct synthesis of few-layer F-doped graphene foam and its lithium/potassium storage properties. ACS Appl. Mater. Interfaces 8, 20682-20690. doi: 10.1021 /acsami.6b04763
Kang, J., Wen, J., Jayaram, S. H., Wang, X., and Chen, S. K. (2013). Electrochemical characterization and equivalent circuit modeling of single-walled carbon nanotube (SWCNT) coated electrodes. J. Power Sources 234, 208-216. doi: 10.1016/j.jpowsour.2013.01.058

Komaba, S., Hasegawa, T., Dahbi, M., and Kubota, K. (2015). Potassium intercalation into graphite to realize high-voltage/high-power potassium-ion batteries and potassium-ion capacitors. Electrochem. Commun. 60, 172-175. doi: 10.1016/j.elecom.2015.09.002

Larcher, D., and Tarascon, J. M. (2015). Towards greener and more sustainable batteries for electrical energy storage. Nat. Chem. 7, 19-29. doi: $10.1038 /$ nchem.2085

Lei, L., Yu, C., Xie, Y., Peng, T., Li, Q., and Yan, C. (2018). Understanding of the ultrastable K-ion storage of carbonaceous anode. Adv. Funct. Mater. 28:1801989. doi: 10.1002/adfm.201801989

Li, Z., Xu, Z., Tan, X., Wang, H., Holt, C. M. B., Stephenson, T., et al. (2013). Mesoporous nitrogen-rich carbons derived from protein for ultra-high capacity battery anodes and supercapacitors. Energy Environ. Sci. 6, 871-878. doi: $10.1039 / \mathrm{c} 2 \mathrm{ee} 23599 \mathrm{~d}$

Lijun, F., Kun, T., Kepeng, S., Van Aken, P. A., Yu, Y., and Maier, J. (2014). Nitrogen doped porous carbon fibres as anode materials for sodium ion batteries with excellent rate performance. Nanoscale 6, 1384-1389. doi: 10.1039/C3NR05374A

Long, Q., Chen, W., Xiong, X., Hu, C., Feng, Z., Hu, P., et al. (2016). Sulfur-doped carbon with enlarged interlayer distance as a high-performance anode material for sodium-ion batteries. Adv. Sci. 2:1500195. doi: 10.1002/advs.201500195

Luo, W., Jian, Z., Xing, Z., Wang, W., Bommier, C., Lerner, M. M., et al. (2015a). Electrochemically expandable soft carbon as anodes for $\mathrm{Na}$-ion batteries. ACS Central Sci. 1, 516-522. doi: 10.1021/acscentsci.5b00329

Luo, W., Wan, J., Ozdemir, B., Bao, W., Chen, Y., Dai, J., et al. (2015b). Potassium ion batteries with graphitic materials. Nano Lett. 15, 7671-7677. doi: 10.1021 /acs.nanolett.5b03667

Ma, C., Shao, X., and Cao, D. (2012). Nitrogen-doped graphene nanosheets as anode materials for lithium ion batteries: a first-principles study. J. Mater. Chem. A 22, 8911-8915. doi: 10.1039/c2jm00166g

Ma, G., Huang, K., Ma, J. S., Ju, Z., Xing, Z., and Zhuang, Q. C. (2017). Phosphorus and oxygen dual-doped graphene as superior anode material for room-temperature potassium-ion batteries. J. Mater. Chem. A 5, 7854-7861. doi: 10.1039/C7TA01108C

Muller, G. A., Cook, J., Kim, H. S., Tolbert, S. H., and Dunn, B. J. N. L. (2015). High performance pseudocapacitor based on 2D layered metal chalcogenide nanocrystals. Nano Lett. 15, 1911-1917. doi: 10.1021/nl504764m

Okoshi, M., Yamada, Y., Komaba, S., Yamada, A., and Nakai, H. (2017). Theoretical analysis of interactions between potassium ions and organic electrolyte solvents: a comparison with lithium, sodium, and magnesium ions. J. Electrochem. Soc. 164, A54-A60. doi: 10.1149/2.0211702jes

Okoshi, M., Yamada, Y., Yamada, A., and Nakai, H. (2013). Theoretical analysis on de-solvation of lithium, sodium, and magnesium cations to organic electrolyte solvents. J. Electrochem. Soc. 160, A2160-A2165. doi: 10.1149/2.074311jes

Sathiya, M., Prakash, A. S., Ramesha, K., Tarascon, J. M., and Shukla, A. K. (2011). V2O5-anchored carbon nanotubes for enhanced electrochemical energy storage. J. Am. Chem. Soc. 133, 16291-16299. doi: 10.1021/ja20 $7285 b$

Shannon, R. (1976). Revised effective ionic radii and systematic studies of interatomic distances in halides and chalcogenides. Acta Crystallogr. Sect. A 32, 751-767. doi: $10.1107 /$ S0567739476001551

Shao, Y., Xiao, J., Wang, W., Engelhard, M., Chen, X., Nie, Z., et al. (2013). Surfacedriven sodium ion energy storage in nanocellular carbon foams. Nano Lett. 13, 3909-3914. doi: 10.1021/nl401995a

Share, K., Cohn, A. P., Carter, R., Rogers, B., and Pint, C. L. (2016). Role of nitrogen doped graphene for improved high capacity potassium ion battery anodes. Acs Nano 10, 9738-9744. doi: 10.1021/acsnano.6b05998

Shin, J. Y., Samuelis, D., and Maier, J. (2011). Sustained lithium-storage performance of hierarchical, nanoporous anatase $\mathrm{TiO}_{2}$ at high rates: emphasis on interfacial storage phenomena. Adv. Funct. Mater. 21, 3464-3472. doi: $10.1002 /$ adfm. 201002527

Su, D., Mcdonagh, A., Qiao, S. Z., and Wang, G. (2016). High-capacity aqueous potassium-ion batteries for large-scale energy storage. Adv. Mater. 29:1604007. doi: 10.1002/adma.201604007 
Suo, L., Borodin, O., Wang, Y., Rong, X., Sun, W., Fan, X., et al. (2017). "Waterin-salt" electrolyte makes aqueous sodium-ion battery safe, green, and longlasting. Adv. Energy Mater. 7:1701189. doi: 10.1002/aenm.201701189

Tarun, B., Aleks, A., Barbara, P., Veronica, B., and Fahlman, B. D. (2010). Enhanced electrochemical lithium storage by graphene nanoribbons. J. Am. Chem. Soc. 132, 12556-12558. doi: 10.1021/ja106162f

Torsten, B., John, W., Tolbert, S. H., and Bruce, D. (2010). Ordered mesoporous alpha- $\mathrm{MoO}_{3}$ with iso-oriented nanocrystalline walls for thin-film pseudocapacitors. Nat. Mater. 9, 146-151. doi: 10.1038/nmat2612

Veronica, A., Jérémy, C., Lowe, M. A., Jong Woung, K., Pierre-Louis, T., Tolbert, S. H., et al. (2013). High-rate electrochemical energy storage through Li+ intercalation pseudocapacitance. Nat. Mater. 12, 518-522. doi: $10.1038 /$ nmat3601

Wang, G., Xiong, X., Xie, D., Lin, Z., and Liu, M. (2018). Chemical activated hollow carbon nanospheres as a high-performance anode material for potassium ion batteries. J. Mater. Chem. A 6, 24317-24323. doi: 10.1039/C8TA09751H

Wang, J., Polleux, J., Lim, J., and Dunn, B. (2007). Pseudocapacitive contributions to electrochemical energy storage in $\mathrm{TiO}_{2}$ (anatase) nanoparticles. J. Phys. Chem. C 111, 14925-14931. doi: 10.1021/jp074464w

Wang, L., Yang, J., Li, J., Chen, T., Chen, S., Wu, Z., et al. (2019). Graphite as a potassium ion battery anode in carbonate-based electrolyte and ether-based electrolyte. J. Power Sources 409, 24-30. doi: 10.1016/j.jpowsour.2018.10.092

Wang, X., Han, K., Qin, D., Li, Q., Wang, C., Niu, C., et al. (2017). Polycrystalline soft carbon semi-hollow microrods as anode for advanced $\mathrm{K}$-ion full batteries. Nanoscale 9, 18216-18222. doi: 10.1039/C7NR06645G

Wang, X., Weng, Q., Liu, X., Wang, X., Tang, D. M., Tian, W., et al. (2014). Atomistic origins of high rate capability and capacity of $\mathrm{N}$-doped graphene for lithium storage. Nano Lett 14, 1164-1171. doi: 10.1021/nl4038592

Wang, X., Xu, X., Niu, C., Meng, J., Huang, M., Liu, X., et al. (2016). Earth abundant $\mathrm{Fe} / \mathrm{Mn}$-based layered oxide interconnected nanowires for advanced K-ion full batteries. Nano Lett. 17, 544-550. doi: 10.1021/acs.nanolett.6b04611

Wang, Y. (2017). Practical challenges in employing graphene for lithium-ion batteries and beyond. Small 1:1700099. doi: 10.1002/smtd.201700099

Wang, Y., Ding, Y., Pan, L., Shi, Y., Yue, Z., Shi, Y., et al. (2016). Understanding the size-dependent sodium storage properties of $\mathrm{Na}_{2} \mathrm{C}_{6} \mathrm{O}_{6}$ based organic electrodes for sodium-ion batteries. Nano Lett. 16, 3329-3334. doi: 10.1021/acs.nanolett.6b00954

Wang, Y., Feng, Z., Yang, S.-Z., Gagnon, C., Gariépy, V., Laul, D., et al. (2018a). Layered oxides- $\mathrm{LiNi}_{1 / 3} \mathrm{Co}_{1 / 3} \mathrm{Mn}_{1 / 3} \mathrm{O}_{2}$ as anode electrode for symmetric rechargeable lithium-ion batteries. J. Power Sources 378, 516-521. doi: 10.1016/j.jpowsour.2017.12.043

Wang, Y., Yang, S., You, Y., Feng, Z., Wen, Z., Gariepy, V., et al. (2018b). A high-capacity and long cycle life aqueous rechargeable lithium-ion battery with $\mathrm{FePO}_{4}$ anode. ACS Appl. Mater. Interfaces 10, 7061-7068. doi: 10.1021/acsami.7b18058

Wang, Z., Selbach, S. M., and Grande, T. (2013). Van der Waals density functional study of the energetics of alkali metal intercalation in graphite. RSC Adv. 4, 4069-4079. doi: 10.1039/C3RA47187J
Wen, Y., He, K., Zhu, Y., Han, F., Xu, Y., Matsuda, I., et al. (2015). Expanded graphite as superior anode for sodium-ion batteries. Nat. Commun. 5:4033. doi: $10.1038 /$ ncomms5033

Wu, L., Buchholz, D., Vaalma, C., Giffin, G. A., and Passerini, S. J. C. (2016). Apple biowaste-derived hard carbon as powerful anode material for $\mathrm{Na}$-ion batteries. ChemElectroChem 3, 292-298. doi: 10.1002/celc.201500437

Wu, X., Leonard, D. P., and Ji, X. (2017). Emerging non-aqueous potassiumion batteries: challenges and opportunities. Chem. Mat. 29, 5031-5042. doi: 10.1021/acs.chemmater.7b01764

Xie, Y., Chen, Y., Liu, L., Tao, P., Fan, M., Xu, N., et al. (2017). Ultrahigh pyridinic $\mathrm{N}$-doped porous carbon monolith enabling high-capacity $\mathrm{K}$ ion battery anodes for both half-cell and full-cell applications. Adv. Mater. 29:1702268. doi: 10.1002/adma.201702268

Xing, Z., Qi, Y., Jian, Z., and Ji, X. (2017). Polynanocrystalline graphite: a new carbon anode with superior cycling performance for K-ion batteries. ACS Appl. Mater. Interfaces 9, 4343-4351. doi: 10.1021/acsami.6b06767

Xu, Y., Zhang, C., Zhou, M., Fu, Q., Zhao, C., Wu, M., et al. (2018). Highly nitrogen doped carbon nanofibers with superior rate capability and cyclability for potassium ion batteries. Nat. Commun. 9:1720. doi: 10.1038/s41467-018-04190-Z

Xu, Z., Liu, J., Chen, C., Potapenko, H., and Wu, M. (2019a). Hydrophilic binder interface interactions inducing inadhesion and capacity collapse in sodium-ion battery. J. Power Sources 427, 62-69. doi: 10.1016/j.jpowsour.2019.04.063

Xu, Z., Wu, M., Chen, Z., Chen, C., Yang, J., Feng, T., et al. (2019b). Direct structure-performance comparison of all-carbon potassium and sodium ion capacitors. Adv. Sci. 6:1802272. doi: 10.1002/advs.201970075

Yang, J., Ju, Z., Jiang, Y., Xing, Z., Xi, B., Feng, J., et al. (2018). Enhanced capacity and rate capability of nitrogen/oxygen dual-doped hard carbon in capacitive potassium-ion storage. Adv. Mater. 30:1700104. doi: 10.1002/adma.2017 00104

Zheng, Y., Wang, Y., Lu, Y., Hu, Y.-S., and Li, J. (2017). A high-performance sodium-ion battery enhanced by macadamia shell derived hard carbon anode. Nano Energy 39, 489-498. doi: 10.1016/j.nanoen.2017.07.018

Zou, X., Xiong, P., Zhao, J., Hu, J., Liu, Z., and Xu, Y. (2017). Recent research progress in non-aqueous potassium-ion batteries. Phys. Chem. Chem. Phys. 19, 26495-26506. doi: 10.1039/C7CP03852F

Conflict of Interest: The authors declare that the research was conducted in the absence of any commercial or financial relationships that could be construed as a potential conflict of interest.

Copyright (c) $2019 \mathrm{Liu}, \mathrm{Xu}, \mathrm{Wu}$, Wang and Karim. This is an open-access article distributed under the terms of the Creative Commons Attribution License (CC BY). The use, distribution or reproduction in other forums is permitted, provided the original author(s) and the copyright owner(s) are credited and that the original publication in this journal is cited, in accordance with accepted academic practice. No use, distribution or reproduction is permitted which does not comply with these terms. 